Casos Clínicos

Arch. Esp. Urol. 2009; 62 (1): 70-73

\section{LEIOMIOSARCOMA DE CORDÓN ESPERMÁTICO. APORTACIÓN DE UN CASO Y REVISIÓN DE LA LITERATURA}

\author{
José Antonio Cánovas Ivorra, José Manuel Sastre \\ Albiach?, Carlos Escrivà Escrivà2, Juan Salvador \\ Fernández García y Antonio Francisco Sánchez Díaz. \\ Servicio de Urología. Servicio de Oncología Médica ${ }^{1}$. \\ Servicio de Cirugía General y Digestiva². Hospital Mare de \\ Deu dels Lliris. Alcoy. Alicante. España.
}

Resumen.- OBJETIVO: Presentación de un nuevo caso de leiomiosarcoma paratesticular.

MÉTODOS: Se describe el caso de un varón de 68 años que consulta por dolor de más de 2 años de evolución en la ingle derecha y cuya exploración física correspondía a la de una hernia inguinal derecha. Durante la herniorrafia se descubre la masa paratesticular.

RESULTADOS: El paciente de somete a orquiectomía radical derecha y tras ello se objetiva una recidiva en la

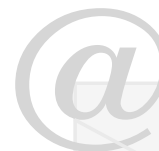

CORRESPONDENCIA

José Antonio Cánovas Ivorra

Urbanización Montiboli 188 A-6

03570 Villajoyosa.Alicante. (España).

jacanovas@hotmail.com

Trabajo recibido: 15 de febrero 2008 cicatriz que se interviene y finalmente una progresión de la enfermedad a nivel locorregional y sistémico.

CONCLUSIONES: Señalamos la escasa frecuencia de este tipo de tumores. Creemos en la orquifuniculectomía como tratamiento de elección. La aplicación de terapias adyuvantes no influye significativamente en la supervivencia de este tipo de pacientes.

Palabras clave: Leiomiosarcoma. Cordón espermático. Tratamiento.

Summary.- OBJECTIVE: To report one new case of paratesticular leiomyosarcoma.

METHODS: We describe the case of a 68-year-old male patient consulting for right groin pain over two years with a physical examination corresponding to right inguinal hernia. During surgery for hernia correction a paratesticular mass was discovered.

RESULTS: Right radical orchiectomy was performed. After the operation there was a recurrence at the scar which was operated and finally the disease progressed both locally and systemically.

CONCLUSIONS: We point out the scarce frequency of this type of tumours. We believe that orchiectomy and excision of the cord is the treatment of choice. The use of adjuvant therapies doesn't influence survival significantly.

Keywords: Leiomyosarcoma. Spermatic cord. Treatment.

\section{INTRODUCCIÓN}

El sarcoma de cordón espermático es una entidad rara (9). Entendemos por estructuras paratesticulares las situadas en relación al testículo. De todos los tumores desarrollados en esta zona, el 7\% son de localización para testicular. Se pueden dividir en líquidos, como hidrocele, varicocele y espermatocele, y sólidos. Estos a su vez se clasifican en benignos como la hernia inguinoescrotal, lipomas, tumor adenomatoide de epidídimo, pseudotumor inflamatorio, cistoadenoma seroso y toda la variedad de tumores mesenquimales benignos; y malignos (8). Los tumores paratesticulares son entidades poco frecuentes, con una incidencia exacta de difícil estimación (7) y de ellos, los sarcomas representan un $90 \%$. Dentro de este grupo, los leiomiosarcoma representan un $10 \%(1,2,3)$. En la infancia el más frecuente es el rabdomiosarcoma y en la edad adulta el liposarcoma, seguido de leiomiosarcoma $(3,7)$. La gran mayoría se desarrollan próximos al testículo y en relación a su polo superior. 
Dada la escasa incidencia no existen series largas de este tipo de patología, por lo que la experiencia en su tratamiento es puntual, aunque lo recomendado es la orquiectomía radical vía inguinal (9).

\section{CASO CLÍNICO}

Paciente varón de 68 años con antecedentes personales de RTU de próstata por hiperplasia benigna de próstata en otro centro, cuyo estudio patológico evidenció la presencia de un adenocarcinoma Gleason 6 con valores de PSA dentro del rango de la normalidad y que posteriormente fue tratado con radioterapia con intención radical encontrándose en la actualidad en intervalo libre de enfermedad.

Consulta por dolor a nivel de ingle derecha de más de 2 años de evolución que aumenta con la bipedestación y disminuye con el decúbito. Diagnosticado de hernia inguinal derecha directa, se interviene practicándose una herniorrafia inguinal y corrección de un hidrocele derecho concomitante. Al tiempo se observa una tumoración carnosa mamelonada adherida al polo superior del testículo de 2 centímetros de diámetro mayor. Se practica orquiectomía radical derecha vía inguinal y exéresis del cordón espermático por encima de anillo inguinal interno. No se evidencia adenopatías. El estudio anatomopatológico evidenció un leiomiosarcoma con positividad para el estudio inmunohistoquímico de Actina y Vimentina y negativo para Antígeno Prostático Específico (Figuras 2 y 3 ).

El estudio de extensión fue normal. Se plantea radioterapia complementaria. A los 9 meses nota la aparición de una lesión excrecente de $3 \times 2.5$ centímetros en la cicatriz de la herniorrafia que se reseca, informándose de recidiva de leiomiosarcoma con actividad mitótica incrementada. No recibe actividad adyuvante. A los 6 meses percibe la aparición de una nueva lesión nodular en flanco derecho con aumento progresivo de tamaño. En el T.A.C. se evidencia un nódulo que infiltra en profundidad la grasa subcutánea y el músculo iliopsoas (Figura 1). Se reseca la tumoración junto a fibras del oblicuo menor, músculo transverso y músculo iliopsoas. El informe patológico es compatible con infiltración por leiomiosarcoma. En ese punto se plantea terapia adyuvante con esquema de adriamicina e ifosfamida por seis ciclos. Tras 12 meses se interviene nuevamente de recidiva local, pétrea al tacto, que atrapa vasos arteriales y venosos de la zona inguinal. El informe concluye recidiva de leiomiosarcoma de alto grado.

La resonancia nuclear magnética practicada evidencia una masa adenopática inguinal con adenopatías retroperitoneales que desestiman toda posibilidad de tratamiento radical. Se decide administrar quimioterapia de segunda línea con esquema de docetaxel y gemcitabina por seis ciclos. Tras 8 meses de intervalo libre de enfermedad se constata la aparición de nueva masa en re- gión inguinal junto a metástasis pulmonares en el T.A.C. de control por lo que se decide tratamiento paliativo con adriamicina. Previamente a esta nueva recidiva el paciente presenta un episodio de hematuria que mediante ecografía demuestra la existencia de una lesión excrecente en pared vesical derecha de 3 centímetros y que se confirma mediante cistoscopia. El paciente desestima la posibilidad de R.T.U. por encontrarse en esquema de administración de quimioterapia y posterior deterioro del estado general.

\section{COMENTARIOS}

El leiomiosarcoma es un tumor paratesticular maligno con una mayor incidencia a partir de la sexta década de la vida $(2,3)$, aunque se describen casos a partir de la cuarta década (1). Suponen el $5 \%$ de todos los sarcomas y el $2 \%$ de todos los tumores urológicos (4).

No se conoce el origen de este tipo de tumores (7), pero se especula con la posibilidad del papel de una degeneración de un leiomioma previo, originado a partir de estructuras funiculares de músculo liso, que puede localizarse en las fibras musculares cremastéricas, en el deferente, en los vasos funiculares, incluso en restos vestigiales de músculos de la pared escrotal, de las fibras conjuntivas del conducto peritoneovaginal o de restos mesoblásticos atrapados en el cordón funicular, dado que en esta compleja región anatómica pueden encontrarse restos epiteliales, mesoteliales y mesenquimales (5). La estimulación hormonal ha sido descrita en la carcinogénesis del leiomiosarcoma (7).

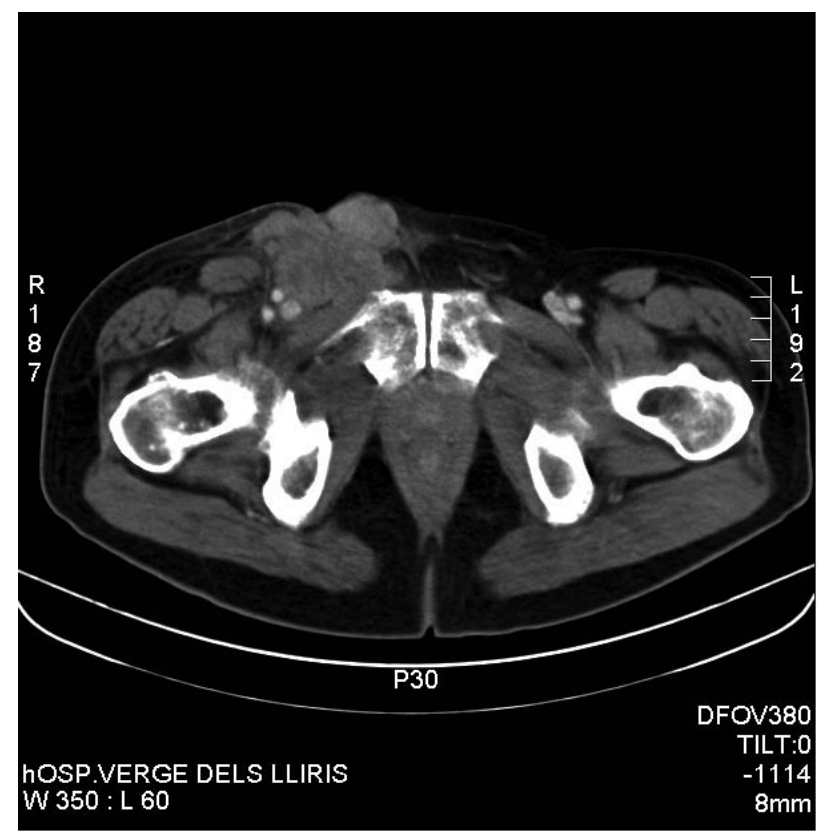

FIGURA 1. Masa de contorno irregular en conducto inguinal derecho con infiltración de musculatura profunda. 
El LMS más frecuente se desarrolla en estructuras funiculares, seguido de escroto y epidídimo (3).

El total de LMS publicados en la literatura oscila en torno a 125 casos, lo que hace que extrapolar actuaciones basadas en estas series de casos sea complejo (3).

Sus manifestaciones clínicas suelen ser inespecíficas, con un predominio del efecto masa local, que se diagnostica tras larga evolución, ya que son indoloros $(3,5)$. Entre sus diagnósticos diferenciales figuran las hernias inguinoescrotales y las adenopatías (3), lesiones inflamatorias, colecciones escrotales inflamatorias y varicoceles (4). Su tamaño en el momento del diagnóstico suele variar entre los 2 y $5 \mathrm{~cm}$. (3). Los estudios analíticos no ofrecen datos dignos de mención, a excepción de algún leiomiosarcoma de cordón espermático productor de B-HCG $(1,3)$.

En cuanto a las técnicas de imagen destacamos el papel del T.A.C. y la ecografía inguinal para confirmar el origen extratesticular del tumor y valorar la afectación local o a distancia (3), pero no para distinguir la naturaleza del tumor (5). La hernia inguinal aparece ecográficamente dependiendo del contenido de la hernia, los quistes epididimarios y el espermatocele aparecen como masas aneicoicas bien definidas, la epididimitis aguda suele aparecer como masa hiperecoica con flujo aumentado en el estudio Doppler, sin embargo, la epididimitis crónica, granulomatosis, tuberculosis, brucelosis, infecciones fúngicas, sarcoidosis o sífilis pueden aparecer como indistinguibles ecográficamente de los tumores (4).

Los hallazgos ecográficos de los tumores extratesticulares son inespecíficos, pero con algunas características que los diferencian. Así, los lipomas suelen aparecer como masas largas, de ecogenicidad variable pero bien definida; los tumores adenomatoides y leiomiomas aparecen como masas de ecogenicidad variable con baja vascularidad y los sarcomas aparecen con ecogenidad heterogénea dependiendo de la diferenciación del tumor, con alta vascularización en el estudio Doppler a diferencia de los tumores benignos (4).

El diagnóstico definitivo viene dado por el estudio anatomopatológico. Las técnicas de inmunohistoquímica si bien facilitan el diagnóstico, en ocasiones pueden no ser concluyentes $(2,5)$. La actina músculo específica debe ser positiva, así como la Desmina y no la Vimentina o Proteína $S 100$, propia de las células adiposas (3).

El diagnóstico diferencial debe considerarse con el rabdomiosarcoma, liposarcoma, histiocitoma maligno fibroso y fibrosarcoma, así como otras lesiones benignas (3). De la fibromatosis puede diferenciarse por su patrón de crecimiento agresivo, pero de otros tumores, como el liposarcoma, puede ser muy difícil su diferenciación en casos de leiomiosarcoma indiferenciado (8). La presencia de necrosis es fundamental para poder plantear tratamiento coadyuvante y establecer un pronóstico, de forma que el grado I no tiene necrosis en la pieza, el grado II, menos del 15\% y el grado III más del 15\% (3). Es el segundo y tercer grado lo más beneficiados por la radioterapia, dado que en grado I la recidiva es menor. La recidiva local es la norma como ocurrió en nuestro caso, y a pesar de tender a permanecer localmente controlados, se han descrito metástasis linfáticas y viscerales en cerebro, hueso y pulmón $(3,9)$, siendo esta la causa de fallecimiento de la tercera parte de los pacientes. La influencia del tamaño tumoral con la recurrencia local no está del todo aclarada (9). Las metástasis ganglionares se harán a retroperitoneo si se originan en el cordón espermático o ganglios inguinales si se localizan en escroto o epidídimo (3). Desde el pun-

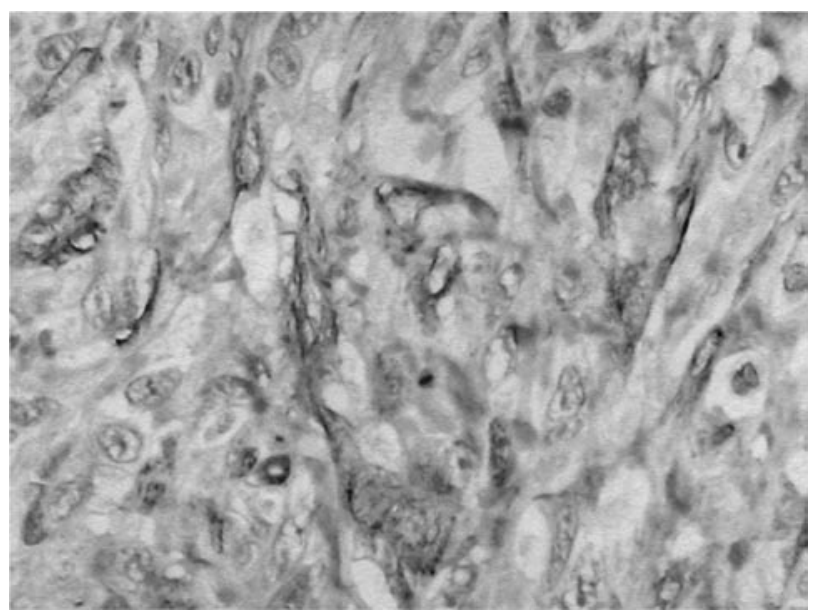

FIGURA 2. Positividad citoplasmática con actina musculoespecífica (HHF x 400).

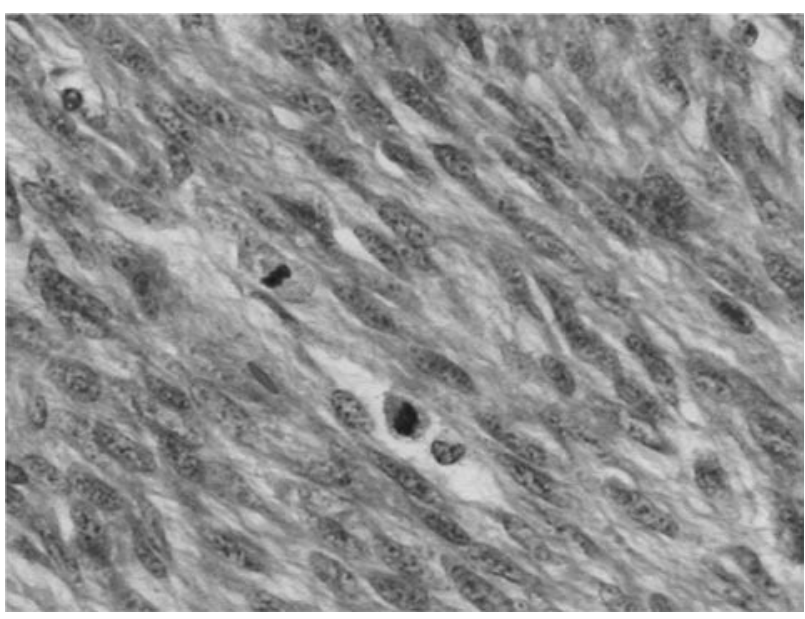

FIGURA 3. Intenso pleomorfismo nuclear y abundantes de mitosis (HE $\times$ 400). 
to de vista anatomopatológico se aceptan como de mal pronóstico: 1) alta celularidad, 2) invasión vascular, 3) áreas necróticas, 4) presencia de células multinucleadas e 5) índice mitótico superior a 2.

Como tratamiento definitivo se acepta la orquiectomía radical vía inguinal con ligadura alta del cordón espermático $(1,2,3,6)$. No existe uniformidad de criterio a la hora de indicar una terapia adyuvante, dada la corta experiencia en su tratamiento (3). Mientras algunos autores defienden la irradiación profiláctica incluso después de la cirugía primaria para controlar la enfermedad local y reducir incluso la recidiva locorregional $(1,2,9)$, otros no creen que tenga papel en el control de recidivas locales. Los defensores de la Radioterapia adyuvante la porponen directa en el canal inguinal y en los tejidos pélvicos adyacentes (9). La linfadenectomía retroperitoneal se reserva para casos de diagnóstico histopatológico de rabdomiosarcoma y casos de extensión locorregional o recidiva $(1,3)$. La mayor parte de las metástasis se hacen vía hematógena, por lo que la irradiación profiláctica carece de indicación en la actualidad (3).

En el seguimiento de este tipo de pacientes está claramente indicada la realización periódica de un T.A.C. La recurrencia de este tipo de patologías es una realidad aceptada, con cifras que pueden oscilar a los 5 años, entre el $10 \%$ y el $60 \%(1,3)$.

\section{DISCUSIÓN}

Los tumores paratesticulares son tumores infrecuentes $y$ con una incidencia exacta difícil de estimar. Se acepta como tratamiento la orquiectomía radical más exéresis de los elementos del cordón. La diseminación puede ser tanto linfática como hematógena mayormente a hígado y pulmón. La linfadenectomía retroperitoneal no está recomendada como profiláctica, y comparado con la cirugía, los efectos de las terapias adyuvantes, incluida la quimioterapia y la radioterapia, son desconocidos, aunque muchos autores la recomiendan a continuación de la cirugía radical (6).

\section{BIBLIOGRAFÍA y LECTURAS RECOMENDADAS ( ${ }^{*}$ lectura de interés $y^{* *}$ lectura fundamental)}

1. Abe DVS, Verona CBM, Suzigan S. Spermatic Cord Leiomyosarcoma. Braz. J. Urol 2001; 27: 569-70.

*2. Cruz Guerra NA, Ramos LC, Montáns Araújo J et al. Leiomiosarcoma paratesticular: descripción de un nuevo caso; Arch. Esp. Urol 2002; 55: 311-4.

**3. Llarena Ibarguren, R., Azurmendi Sastre, V., Martín Bazaco, J. et al. Leiomiosarcoma paratesticular. Revisión y puesta al día. Arch. Esp. de Urol 2004; 57: 525-30.

*4. Secil M, Kefi A, Gulbahar F et al. Sonographic Features of Spermatic Cord Leiomyosarcoma. J. Ultrasound
Med 2004; 23:973-76.

5. Ptochos A, Iosifidis N, Papazafiriou G et al. Primary Paratesticular Epithelioid Leiomyosarcoma. Urol 2003; Int., 70: 321-23.

6. Shih-Ming O, Shang-Sen L, Yi-Jen P et al. Production of Beta-H.C.G. by Spermatic Cord Leiomyosarcoma: A Paraneoplasic Syndrome?; J. Androl 2006; 27: 643-44.

*7. Eslami Varzaneh F, Verghese M, Shmookler B. Paratesticular leiomyosarcoma in an elderly man; Urology; 60 (6): 1112xxi-1112xxiii, 2002.

8. Fisher C, Goldblum J, Epstein J et al. Leiomyosarcoma of the Paratesticular Region. Am. J. Surg. Pathol 2001; 25: 1143-49.

*9. Ballo MT, Zagars GK, Pisters PWT et al. Spermatic cord sarcoma: Outcome, patterns of failure and management. J. Urol 2001; 166: 1306-10. 